\title{
Correction to: Microbiome definition re- visited: old concepts and new challenges
}

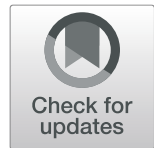

\author{
Gabriele Berg ${ }^{1 * \dagger}$, Daria Rybakova ${ }^{1 \dagger}$, Doreen Fischer ${ }^{2 \dagger}$, Tomislav Cernava $^{1}$, Marie-Christine Champomier Vergès ${ }^{3}$, \\ Trevor Charles ${ }^{4,5}$, Xiaoyulong Chen ${ }^{6}$, Luca Cocolin ${ }^{7}$, Kellye Eversole $^{8+}$, Gema Herrero Corral $^{9}$, Maria Kazou ${ }^{10}$, \\ Linda Kinkel $^{11 \dagger}$, Lene Lange ${ }^{12}$, Nelson Lima ${ }^{13}$, Alexander Loy ${ }^{14}$, James A. Macklin ${ }^{15}$, Emmanuelle Maguin ${ }^{3}$, \\ Tim Mauchline ${ }^{16}$, Ryan McClure ${ }^{17}$, Birgit Mitter ${ }^{18}$, Matthew Ryan ${ }^{19}$, Inga Sarand ${ }^{20}$, Hauke Smidt ${ }^{21}$, Bettina Schelkle ${ }^{7}$, \\ Hugo Roume ${ }^{22}$, G. Seghal Kiran ${ }^{23}$, Joseph Selvin ${ }^{24}$, Rafael Soares Correa de Souza ${ }^{25}$, Leo van Overbeek ${ }^{21}$, \\ Brajesh K. Singh ${ }^{26,27}$, Michael Wagner ${ }^{14 \dagger}$, Aaron Walsh ${ }^{28}$, Angela Sessitsch ${ }^{18 \dagger}$ and Michael Schloter ${ }^{2^{*}+}$
}

\section{Correction to: Microbiome 8, 103 (2020) https://doi.org/10.1186/s40168-020-00875-0}

Following publication of the original article [1], an error was identified in the affiliation of the 8th author, Luca Cocolin.

The incorrect affiliation is: European Food Information Council, Brussels, Belgium

The correct affiliation is: Department of Agricultural, Forest and Food Sciences, University of Turin, Italy

The affiliation has been updated in this correction article.

\section{Author details \\ 'Institute of Environmental Biotechnology, Graz University of Technology, Graz, Austria. ${ }^{2}$ Helmholtz Zentrum München, Oberschleissheim, Germany. ${ }^{3}$ MICALIS, INRA, AgroParisTech, Université Paris-Saclay, 78350 Jouy-en-Josas, France. ${ }^{4}$ Waterloo Centre for Microbial Research, University of Waterloo, 200 University Avenue West, Waterloo, ON N2L 3G1, Canada. ${ }^{5}$ Metagenom Bio, 550 Parkside Drive, Unit A9, Waterloo, ON N2L 5 V4, Canada. ${ }^{6}$ Guizhou Provincial Key Laboratory for Agricultural Pest Management of the Mountainous Region, Guizhou University, Guiyang 550025, Guizhou, China. 7Department of Agricultural, Forest and Food Sciences, University of Turin, Turin, Italy. ${ }^{8}$ International Alliance for Phytobiomes Research, Lee's Summit, MO, USA. ${ }^{9}$ MICA, INRA, 78350 Jouy-en-Josas, France. ${ }^{10}$ Laboratory of Dairy}

The original article can be found online at https://doi.org/10.1186/s40168020-00875-0

*Correspondence: gabriele.berg@tugraz.at; schloter@helmholtzmuenchen.de ${ }^{\dagger}$ Gabriele Berg, Daria Rybakova, Doreen Fischer, Kellye Eversole, Linda Kinkel, Michael Wagner, Angela Sessitsch and Michael Schloter contributed equally to this work.

${ }^{1}$ Institute of Environmental Biotechnology, Graz University of Technology,

Graz, Austria

${ }^{2}$ Helmholtz Zentrum München, Oberschleissheim, Germany

Full list of author information is available at the end of the article
Research, Department of Food Science and Human Nutrition, Agricultural University of Athens, Athens, Greece. ${ }^{11}$ Department of Plant Pathology, University of Minnesota, St. Paul, MN 55108, USA. ${ }^{12}$ BioEconomy, Research, \& Advisory, Valby, Denmark. ${ }^{13}$ CEB-Centre of Biological Engineering, University of Minho, Campus de Gualtar, 4710-057 Braga, Portugal. ${ }^{14}$ Department of Microbial Ecology and Ecosystem Science, University of Vienna, Vienna, Austria. ${ }^{15}$ Agriculture and Agri-Food Canada, Ottawa, Canada. ${ }^{16}$ Sustainable Agriculture Sciences, Rothamsted Research, Harpenden, UK. ${ }^{17}$ Biological Sciences Division, Pacific Northwest National Laboratory, Richland, WA 99352, USA. ${ }^{18}$ Bioresources Unit, AIT Austrian Institute of Technology, Tulln, Austria.

${ }^{19} \mathrm{CABI}$, Bakeham Lane, Egham, Surrey TW20 9TY, UK. ${ }^{20}$ Department of Chemistry and Biotechnology, Tallinn University of Technology, Tallinn, Estonia. ${ }^{21}$ Laboratory of Microbiology, Wageningen University \& Research, Wageningen, the Netherlands. ${ }^{22}$ MGP, INRA, 78350 Jouy-en-Josas, France. ${ }^{23}$ Dept of Food Science and Technology, Pondicherry University, Puducherry, India. ${ }^{24}$ Department of Microbiology, Pondicherry University, Puducherry, India. ${ }^{25}$ Genomics for Climate Change Research Center (GCCRC),

Universidade Estadual de Campinas (UNICAMP), Campinas, SP, Brazil. ${ }^{26}$ Hawkesbury Institute for the Environment, Western Sydney University, Penrith, NSW, Australia. ${ }^{27} \mathrm{Global}$ Centre for Land-Based Innovation, Western Sydney University, Penrith, NSW, Australia. ${ }^{28}$ Teagasc Food Research Centre, Moorepark, Fermoy, Co. Cork, Ireland.

Published online: 20 August 2020

\section{Reference}

1. Berg G, Rybakova D, Fischer F, Cernava T, Vergès M-CC, Charles T, et al. Microbiome definition re-visited: old concepts and new challenges. Microbiome. 2020;8:103. https://doi.org/10.1186/s40168-020-00875-0.

C C The Author(s). 2020 Open Access This article is licensed under a Creative Commons Attribution 4.0 International License, which permits use, sharing, adaptation, distribution and reproduction in any medium or format, as long as you give appropriate credit to the original author(s) and the source, provide a link to the Creative Commons licence, and indicate if changes were made. The images or other third party material in this article are included in the article's Creative Commons licence, unless indicated otherwise in a credit line to the material. If material is not included in the article's Creative Commons licence and your intended use is not permitted by statutory regulation or exceeds the permitted use, you will need to obtain permission directly from the copyright holder. To view a copy of this licence, visit http://creativecommons.org/licenses/by/4.0/ The Creative Commons Public Domain Dedication waiver (http://creativecommons.org/publicdomain/zero/1.0/) applies to the data made available in this article, unless otherwise stated in a credit line to the data. 